\title{
TEMPO DE ALEITAMENTO MATERNO EXCLUSIVO EM RECÉM-NASCIDOS PREMATUROS E A TERMO
}

\section{Time of exclusive breastfeeding of preterm and term newborn babies}

\author{
Waléria Ferreira da Silva ${ }^{(1)}$, Zelita Caldeira Ferreira Guedes ${ }^{(2)}$
}

\section{RESUMO}

Objetivos: determinar o tempo de aleitamento materno exclusivo de recém nascidos prematuros e observar se estes apresentam um índice de aleitamento materno exclusivo diferente do apresentado pelo município de Maceió, na I e II Pesquisa de Prevalência de Aleitamento Materno nas Capitais Brasileiras e no Distrito Federal. Além de comparar o tempo de aleitamento materno e aleitamento materno exclusivo de recém nascidos prematuros e a termo e verificar as causas do desmame precoce e suas consequências. Método: trata-se de uma investigação de campo, de caráter exploratório e longitudinal da prevalência do aleitamento materno exclusivo de recém nascidos prematuro e à termo. Resultados: o percentual de aleitamento materno e de aleitamento materno exclusivo de prematuros aos 6 meses. Houve diferença significante para a variável peso ao nascimento. Dentre as causas do desmame precoce, os fatores educacionais seguidos dos fatores culturais apareceram em maior porcentagem. Como consequência do desmame precoce a prática de hábitos orais ocorreu em $98,1 \%$ das crianças. Conclusão: as crianças prematuras permaneceram mais tempo em aleitamento materno e em aleitamento materno exclusivo que as nascidas a termo. Os fatores educacionais e culturais foram as principais causas do desmame precoce e sua consequência foi o uso de chupeta e mamadeira.

DESCRITORES: Aleitamento Materno; Desmame; Recém-Nascido

\section{INTRODUÇÃO}

Os mamíferos são animais dotados de glândulas mamárias e nas fêmeas desta espécie estas glândulas secretam leite, alimento indispensável aos mamíferos de mais tenra idade. Também, todo mamífero já nasce dotado de um instinto natural, o de mamar. Contudo, mães precisam aprender a amamentar e os bebês a serem amamentados.

A amamentação é o ato mais natural e o melhor alimento para o bebê devido aos benefícios

(1) Fonoaudióloga da Secretaria Municipal de Saúde de Maceió e da Secretaria Estadual de Educação de Alagoas, Mestrado em Distúrbios da Comunicação Humana pela Universidade Federal de São Paulo, UNIFESP, São Paulo, São Paulo, Brasil; Especialista em Motricidade Orofacial com ênfase em Disfagia.

(2) Fonoaudióloga; Professora Doutora Associada do Departamento de Fonoaudiologia da UNIFESP, Universidade Federal de São Paulo, São Paulo, São Paulo, Brasil; Pós-Doutorado realizado na Universidade René Descartes, Paris 5, França.

Conflito de interesses: inexistente nutricionais, emocionais e econômicos. Além disso, ela promove estímulos neurais que favorecem um adequado crescimento e desenvolvimento facial favorecendo as estruturas e funções estomatognáticas, prevenindo maloclusões por hipodesenvolvimento, devendo o fonoaudiólogo ser um dos incentivadores desta prática ${ }^{1}$. Porém, esta é ainda uma prática realizada por uma minoria de mulheres, que a abandonam precocemente, introduzindo alimentos complementares à dieta da criança, não seguindo a orientação da Organização Mundial de Saúde (OMS), que recomenda o aleitamento materno exclusivo até 6 meses de idade e complementado até 2 anos ${ }^{2}$.

O aleitamento materno traz benefícios ímpares, principalmente para prematuros, levando a maiores índices de inteligência e de acuidade visual; melhora do sistema de defesa, devido a grande oferta de imunoglobulinas; promove maior proteção contra infecções, flatulência, diarréia ou constipação; confere melhor digestibilidade e ausência de fatores alergênicos; diminui o risco de falência 
respiratória, apnéia e displasia broncopulmonar; reduz o risco de obesidade ${ }^{3}$; favorece uma melhor mobilidade, tonicidade e postura dos órgãos fonoarticulatórios, devido ao esforço para conseguir sugar o leite do peito materno; promove uma satisfação oral máxima ao RN, além de possibilitar estímulos táteis, visuais, auditivos, base para o desenvolvimento emocional, perceptivo, motor, cognitivo e físico; reduz o risco de câncer de mama e de ovário materno; promove a contração do útero e a perda de peso natural da mulher ${ }^{4}$.

Pesquisas revelam pouco sucesso do aleitamento materno exclusivo em recém-nascidos prematuros devido às barreiras hospitalares, à imaturidade fisiológica e neurológica do prematuro, à falta de orientação e à insegurança materna em lidar com seu filho, hipotonia muscular, hiper-reatividade aos estímulos do meio ambiente, inadequação das funções de sucção-respiração-deglutição, fatores culturais (uso de chupeta e mamadeira) e crenças, a promoção comercial de fórmulas lácteas infantis, o trabalho materno fora do lar, a falta de informação a respeito do aleitamento materno e as práticas inadequadas dos profissionais de saúde 5 . Um número grande de prematuros que se encontram internados em Unidades de Terapias Intensivas Neonatais não recebem leite de sua própria mãe, sendo estes privados da melhor possibilidade de nutrição e proteção ${ }^{6}$.

O aleitamento materno exclusivo sofre variações significantes em suas taxas de acordo com o local e as características socioeconomicas e culturais da população estudada, uma vez que estes têm influenciado a lactante, determinando por quanto tempo irá amamentar ${ }^{7}$. Segundo dados da I Pesquisa de Prevalência de Aleitamento Materno nas Capitais Brasileiras e no Distrito Federal (I PPAM-CDF/1999), Maceió tem uma média de aleitamento materno de 5,7 meses e uma média aleitamento materno exclusivo de 8 dias, sendo a capital brasileira com os menores índices de aleitamento materno ${ }^{8}$. A pesquisa supracitada teve como um de seus objetivos estimar, para a área urbana das capitais, regiões brasileiras e Brasil a prevalência e a duração mediana do aleitamento materno e do aleitamento materno exclusivo. Diante do exposto, a hipótese desta pesquisa é que os bebês prematuros permanecem em aleitamento materno exclusivo por menos de seis meses.

Partindo deste pressuposto, a presente pesquisa tem por objetivo analisar o tempo de aleitamento materno exclusivo de recém nascidos prematuros e a termo e verificar os fatores associados ao desmame precoce.

\section{MÉTODO}

Participaram do estudo 93 mães de crianças prematuras que nasceram na Maternidade Escola Santa Mônica (maternidade especializada no atendimento a gestantes de risco no município de Maceió, sendo esta a única maternidade no município a praticar o Método Mãe-Canguru) e 93 mães de crianças nascidas a termo dos postos de referência de cada distrito de saúde do Município de Maceió (Maceió possui sete distritos de saúde e cada um conta com uma unidade polo). Para distribuir o número de mães que seriam entrevistadas em cada Unidade Básica de Saúde foi realizado o cálculo estratificado de acordo com a quantidade de postos de cada distrito de saúde com a finalidade de subdividir a amostra nas 7 Unidades Básicas de Saúde de referência de cada Distrito de Saúde. Em ambos os grupos as crianças tinham no máximo 1 ano de idade, selecionadas por meio da técnica de amostragem sistemática, onde a cada mãe que se enquadrava nos critérios de inclusão e exclusão, uma era descartada.

Porém, das 93 crianças nacidas prematuras foram analisados 82 questionários, pois 3 mães não compareceram aos retornos nem foi possível contatar as mesmas e outras 8 mães tinham crianças gemelares, sendo necessário desconsiderar uma das crianças por meio de sorteio para não gerar um erro estatístico de duplicidade de dados. Com relação aos recém nascidos termo, foram analisados 90 questionários, pois 3 mães não foi possível contatá-las.

Os critérios de inclusão dos sujeitos na pesquisa foram mães de recém-nascidos prematuros e mães de recém-nascidos a termo com até um ano de idade. E os critérios de exclusão da pesquisa foram mães de crianças com diagnóstico de síndromes genéticas, com malformações motoras orais e/ou congênitas ou com problemas neurológicos previamente diagnosticados.

A pesquisa caracteriza-se por ser uma investigação de campo, de caráter exploratório e longitudinal da ocorrência do aleitamento materno exclusivo de recém nascidos prematuros e a termo, onde foi aplicado um questionário padronizado com as mães desses bebês. O formulário de coleta de dados foi dividido em dados de identificação do bebê e de sua mãe, experiência com a amamentação, causas do desmame precoce foram categorizadas (educacionais, socieconômicas, culturais e anatomofisiológicas) para facilitar a discussão, como também as consequências do desmame precoce (diarréia, pneumonia, infecções e hábitos orais).

Para esta pesquisa foi realizado o cálculo do tamanho da amostra para uma proporção de 
população finita, realizado em uma calculadora eletrônica, disponível no site http://lia.uncisal.edu. br/ensino/pdf2/CTA_Media_finita.xls, cujo valor da população $(\mathrm{N})$ considerado foi 2659 crianças, valor este obtido no site do DataSUS para o número de crianças prematuras nascidas em Maceió no ano de 2008. Para as crianças a termo foi adotada o mesmo valor de $\mathrm{n}$ obtido no cálculo para crianças prematuras. $O$ valor estimado para a variável reduzida (Z) foi de 1,96 para um valor de alfa igual a 0,05 e a proporção $(p)$ neste estudo foi de 0,5 e o erro tolerável considerado (E) foi igual a 0,1. O intervalo de confiança considerado foi de $95 \%$.

A amostra foi coletada num período de agosto de 2008 à novembro de 2009. As mães de crianças com menos de 6 (seis) meses de idade no momento da pesquisa, foram acompanhadas mensalmente pela fonoaudióloga pesquisadora do presente estudo até seus filhos completarem tal idade ou até realizarem o desmame precoce, com a finalidade de verificar o tempo de aleitamento materno exclusivo. Durante a pesquisa, 47 mães de crianças nascidas prematuras foram acompanhadas uma vez que as mesmas ainda permaneciam amamentando seus filhos, como também para obtermos dados mais exatos e 35 foi realizada apenas a entrevista, pois já haviam realizado o desmame precoce, podendo haver variaçção quanto ao tempo real de desmame. Já as mães de crianças nascidas a termo 29 foram acompanhadas e 61 responderam apenas a entrevista, uma vez que a maioria delas já havia realizado o desmame precoce de seus filhos.

$\mathrm{Na}$ estatística descritiva, os dados categóricos foram resumidos por meio de frequência absoluta (N) e relativa (\%) ao total de pacientes em cada grupo estudado.

A análise descritiva foi realizada por meio dos dados de locação, cujas medidas utilizadas foram a média, a moda e a mediana e também pelos dados de dispersão absoluta, sendo utilizadas as medidas da variância e do desvio padrão.

O presente estudo foi encaminhado para avaliação do Comitê de Ética em Pesquisa da Universidade Estadual de Ciências da Saúde de Alagoas sob o protocolo № 745/07 e da Universidade Federal de São Paulo 1226/09.

$\mathrm{Na}$ estatística analítica, os dados numéricos foram comparados com o teste $\mathrm{t}$ para amostras independentes, ou pelo teste não paramétrico de Mann-Whitney. Para validar as correlações, utilizamos o Teste de Correlação e para verificar o grau de relação do tempo de amamentação com todas as variáveis quantitativas foi utilizado o teste de Correlação de Spearman. Por último, foram avaliadas as causas do desmame precoce e suas possíveis consequências.

A significância estatística foi estabelecida para valores de $p<0,05$. Todas as análises e gráficos foram realizados com a utilização do software estatístico Minitab, versão 15.1.

\section{RESULTADOS}

Para facilitar a compreensão os resultados foram divididos em dados de correlação do aleitamento materno exclusivo entre RNPT e RNT e as variáveis: peso ao nascer, idade gestacional, apgar no $5^{\prime}$, número de consultas de pré natal, idade mãe, escolaridade, tempo UTI, tempo berçário intermediário, tempo alojamento conjunto, tempo internação da criança, tempo de uso da sonda para alimentação, número de sessões de fonoterapia, tempo de oxihood, tempo de uso de CPAP, tempo de uso de tubo orotraqueal, temp de uso de oxigênio. Analisou-se o tempo de aleitamento materno e aleitamento materno exclusivo de RNPT e RNT, as causa e as consequências do desmame precoce. 
Tabela 1 - Correlação do tempo de aleitamento materno exclusivo de crianças prematuras e a termo com as variáveis quantitativas

\begin{tabular}{ccccccc}
\hline \multirow{2}{*}{ Variáveis } & \multicolumn{2}{c}{$\begin{array}{c}\text { Tempo AME } \\
\text { RNPT }\end{array}$} & \multicolumn{2}{c}{$\begin{array}{c}\text { Tempo AME } \\
\text { RNT }\end{array}$} & \multicolumn{2}{c}{$\begin{array}{c}\text { Tempo AME } \\
\text { total }\end{array}$} \\
\cline { 2 - 6 } & Corr & p-valor & Corr & p-valor & Corr & p-valor \\
\hline Peso ao nascer & $-0,7 \%$ & 0,951 & $-7,1 \%$ & 0,504 & $-17,7 \%$ & 0,020 \\
Idade Gestacional & $-5,2 \%$ & 0,642 & $-\mathrm{x}-$ & $-\mathrm{x}-$ & $-5,2 \%$ & 0,642 \\
Apgar no 5' & $3,7 \%$ & 0,747 & $-\mathrm{x}-$ & $-\mathrm{x}-$ & $3,7 \%$ & 0,747 \\
n consultas & $-0,5 \%$ & 0,967 & $0,3 \%$ & 0,974 & $-7,4 \%$ & 0,338 \\
Idade mae & $-18,8 \%$ & 0,091 & $17,1 \%$ & 0,107 & $-0,6 \%$ & 0,940 \\
Escolaridade & $9,1 \%$ & 0,415 & $-9,8 \%$ & 0,359 & $-1,8 \%$ & 0,818 \\
Tempo UTI & $10,2 \%$ & 0,363 & $-\mathrm{x}-$ & $-\mathrm{x}-$ & $10,2 \%$ & 0,363 \\
Tempo BI & $-17,0 \%$ & 0,127 & $-\mathrm{x}-$ & $-\mathrm{x}-$ & $-17,0 \%$ & 0,127 \\
Tempo AC & $-12,6 \%$ & 0,258 & $-\mathrm{x}-$ & $-\mathrm{x}-$ & $-12,6 \%$ & 0,258 \\
Tempo internação cça & $-8,9 \%$ & 0,424 & $-\mathrm{x}-$ & $-\mathrm{x}-$ & $-8,9 \%$ & 0,424 \\
Tempo uso sonda & $-1,0 \%$ & 0,932 & $-\mathrm{x}-$ & $-\mathrm{x}-$ & $-1,0 \%$ & 0,932 \\
n sessoes fono & $-16,4 \%$ & 0,141 & $-\mathrm{x}-$ & $-\mathrm{x}-$ & $-16,4 \%$ & 0,141 \\
Tempo Oxihood & $-10,7 \%$ & 0,340 & $-\mathrm{x}-$ & $-\mathrm{x}-$ & $-10,7 \%$ & 0,340 \\
Tempo CPAP & $-6,4 \%$ & 0,571 & $-\mathrm{x}-$ & $-\mathrm{x}-$ & $-6,4 \%$ & 0,571 \\
Tempo tubo & $7,9 \%$ & 0,482 & $-\mathrm{x}-$ & $-\mathrm{x}-$ & $7,9 \%$ & 0,482 \\
Temp O2 & $-4,8 \%$ & 0,673 & $-\mathrm{x}-$ & $-\mathrm{x}-$ & $-4,8 \%$ & 0,673 \\
\hline
\end{tabular}

O peso ao nascimento das crianças prematuras foi menor que o das crianças nascidas a termo, onde a média no grupo de prematuros foi de $1481 \mathrm{~g}$, variando de 680 até $1995 \mathrm{~g}$, e a média no grupo de crianças nascidas a termo foi de $3313 \mathrm{~g}$, variando de 2300 até $4500 \mathrm{~g}$.
A correlação negativa implica que as variáveis são inversamente proporcionais, ou seja, a medida que uma o peso ao nascer aumenta o tempo de aleitamento materno exclusivo decresce.

Tabela 2 - Distribuição de frequências do tempo de aleitamento materno

\begin{tabular}{lcccc}
\hline $\begin{array}{l}\text { Tempo de aleitamento } \\
\text { materno (dias) }\end{array}$ & $\begin{array}{c}\text { RNs } \\
\text { N (\%) }\end{array}$ & $\begin{array}{c}\text { RNPT } \\
\text { N (\%) }\end{array}$ & $\begin{array}{c}\text { RNT } \\
\text { N (\%) }\end{array}$ & $\mathbf{p}^{(1)}$ \\
\hline 0 (zero) & $11(6,4 \%)$ & $3(3,7 \%)$ & $8(8,9 \%)$ & \\
De 1 a 30 & $7(4,1 \%)$ & - & $7(7,8 \%)$ & \\
De 31 a 60 & $8(4,6 \%)$ & $3(3,7 \%)$ & $6(6,7 \%)$ & \\
De 61 a 90 & $12(7,0 \%)$ & $4(4,9 \%)$ & $8(8,9 \%)$ & 0,003 \\
De 91 a 120 & $15(8,7 \%)$ & $6(7,3 \%)$ & $9(10,0 \%)$ & \\
De 121 a 150 & $4(2,3 \%)$ & $2(2,4 \%)$ & $2(2,2 \%)$ & \\
De 151 a 179 & - & - & - & \\
180 dias e mais & $115(66,9 \%)$ & $64(78,0 \%)$ & $50(55,5 \%)$ & \\
\hline
\end{tabular}

(1) nível de significância do teste de Mann-Whitney 
Tabela 3 - Distribuição de frequências do tempo de aleitamento materno exclusivo

\begin{tabular}{lcccc}
\hline $\begin{array}{l}\text { Tempo de aleitamento } \\
\text { materno exclusivo (dias) }\end{array}$ & $\begin{array}{c}\text { RNs } \\
\text { N (\%) }\end{array}$ & $\begin{array}{c}\text { RNPT } \\
\text { N (\%) }\end{array}$ & $\begin{array}{c}\text { RNT } \\
\text { N (\%) }\end{array}$ & p $^{(1)}$ \\
\hline 0 (zero) & $21(12,2 \%)$ & $10(12,1 \%)$ & $11(12,2 \%)$ & \\
De 1 a 30 & $20(11,6 \%)$ & $3(3,7 \%)$ & $17(19,0 \%)$ & \\
De 31 a 60 & $20(11,6 \%)$ & $9(11,0 \%)$ & $11(12,2 \%)$ & \\
De 61 a 90 & $15(8,7 \%)$ & $6(7,3 \%)$ & $9(10,0 \%)$ & 0,023 \\
De 91 a 120 & $21(12,2 \%)$ & $13(15,9 \%)$ & $8(8,9 \%)$ & \\
De 121 a 150 & $8(4,7 \%)$ & $4(4,9 \%)$ & $4(4,4 \%)$ & \\
De 151 a 179 & $1(0,6 \%)$ & $1(1,2 \%)$ & - & \\
180 dias e mais & $66(38,4 \%)$ & $36(43,9 \%)$ & $30(33,3 \%)$ & \\
\hline
\end{tabular}

(1) nível de significância do teste de Mann-Whitney

Das crianças estudadas, $61,6 \%$ foram desmamadas com menos de 180 dias.

Tabela 4 - Compara as causas do desmame precoce devido a fatores educacionais para tempo aleitamento materno exclusivo por grupo

\begin{tabular}{cccccccccc}
\hline $\begin{array}{c}\text { Causas Desmame } \\
\text { Fatores } \\
\text { Educacionais }\end{array}$ & Média & Mediana & $\begin{array}{c}\text { Desvio } \\
\text { Padrão }\end{array}$ & Q1 & Q3 & N & IC & ${ }^{(1)}$ p-valor \\
\hline \multirow{2}{*}{ RNPT } & Não & 163,6 & 180 & 41,9 & 173 & 180 & 44 & 12,4 & \\
& Sim & 74,9 & 90 & 57,9 & 6 & 120 & 38 & 18,4 & $<0,001$ \\
& Sim & 63,0 & 49 & 65,5 & 9 & 105 & 6 & 52,4 & \\
\hline \multirow{2}{*}{ RNT } & Não & 151,7 & 180 & 47,1 & 120 & 180 & 38 & 15,0 & $<0,001$ \\
& Sim & 56,9 & 40 & 47,8 & 23 & 90 & 52 & 13,0 & $<$ \\
\multirow{2}{*}{ Total } & Não & 158,1 & 180 & 44,5 & 128 & 180 & 82 & 9,6 & $<0,001$ \\
\hline
\end{tabular}

(1) nível de significância do teste de Mann-Whitney

Tabela 5 - Compara desmame fatores culturais para o tempo de aleitamento materno exclusivo por grupo

\begin{tabular}{cccccccccc}
\hline $\begin{array}{c}\text { Desmame } \\
\text { Fatores } \\
\text { Culturais }\end{array}$ & Média & Mediana & $\begin{array}{c}\text { Desvio } \\
\text { Padrão }\end{array}$ & Q1 & Q3 & N & IC & ${ }^{(1)}$ p-valor \\
\hline \multirow{2}{*}{ MESM } & Não & 148,4 & 180 & 59,1 & 120 & 180 & 54 & 15,8 & \\
& Sim & 72,4 & 75 & 50,2 & 35 & 114 & 28 & 18,6 & $<0,001$ \\
& Sim & 54,3 & 49 & 50,3 & 28 & 75 & 4 & 49,3 & \\
\hline \multirow{2}{*}{ UBS } & Não & 164,2 & 180 & 35,6 & 180 & 180 & 32 & 12,3 & $<0,001$ \\
& Sim & 59,9 & 49 & 48,2 & 26 & 90 & 58 & 12,4 & $<$ \\
\multirow{2}{*}{ Total } & Não & 154,3 & 180 & 51,9 & 128 & 180 & 86 & 11,0 & $<0,001$ \\
\hline
\end{tabular}

(1) nível de significância do teste de Mann-Whitney 
Tabela 6 - Compara as consequências do desmame para o tempo de aleitamento materno exclusivo por grupo

\begin{tabular}{cccccccccc}
\hline $\begin{array}{c}\text { Consequencias } \\
\text { desmame }\end{array}$ & Média & Mediana & $\begin{array}{c}\text { Desvio } \\
\text { Padrão }\end{array}$ & Q1 & Q3 & N & IC & ${ }^{(1)}$ p-valor \\
\hline \multirow{2}{*}{ MESM } & Não & 176,8 & 180 & 28,3 & 180 & 180 & 37 & 9,1 & \\
& Sim & 77,8 & 90 & 54,7 & 30 & 120 & 45 & 16,0 & $<0,001$ \\
& Sim & 70,0 & 60 & 62,6 & 19 & 116 & 7 & 46,4 & \\
\hline \multirow{2}{*}{ UBS } & Não & 166,5 & 180 & 33,4 & 180 & 180 & 31 & 11,8 & $<0,001$ \\
& Sim & 60,4 & 52 & 48,0 & 27 & 90 & 59 & 12,2 & $<$ \\
\multirow{2}{*}{ Total } & Não & 172,1 & 180 & 30,9 & 180 & 180 & 68 & 7,4 & $<0,001$ \\
& Sim & 67,9 & 60 & 51,5 & 27 & 116 & 104 & 9,9 & \\
\hline
\end{tabular}

(1) nível de significância do teste de Mann-Whitney

Os fatores socioeconômicos e anatomofisiológicos não apresentaram significância estatística quando comparados ao tempo de aleitamento materno exclusivo.

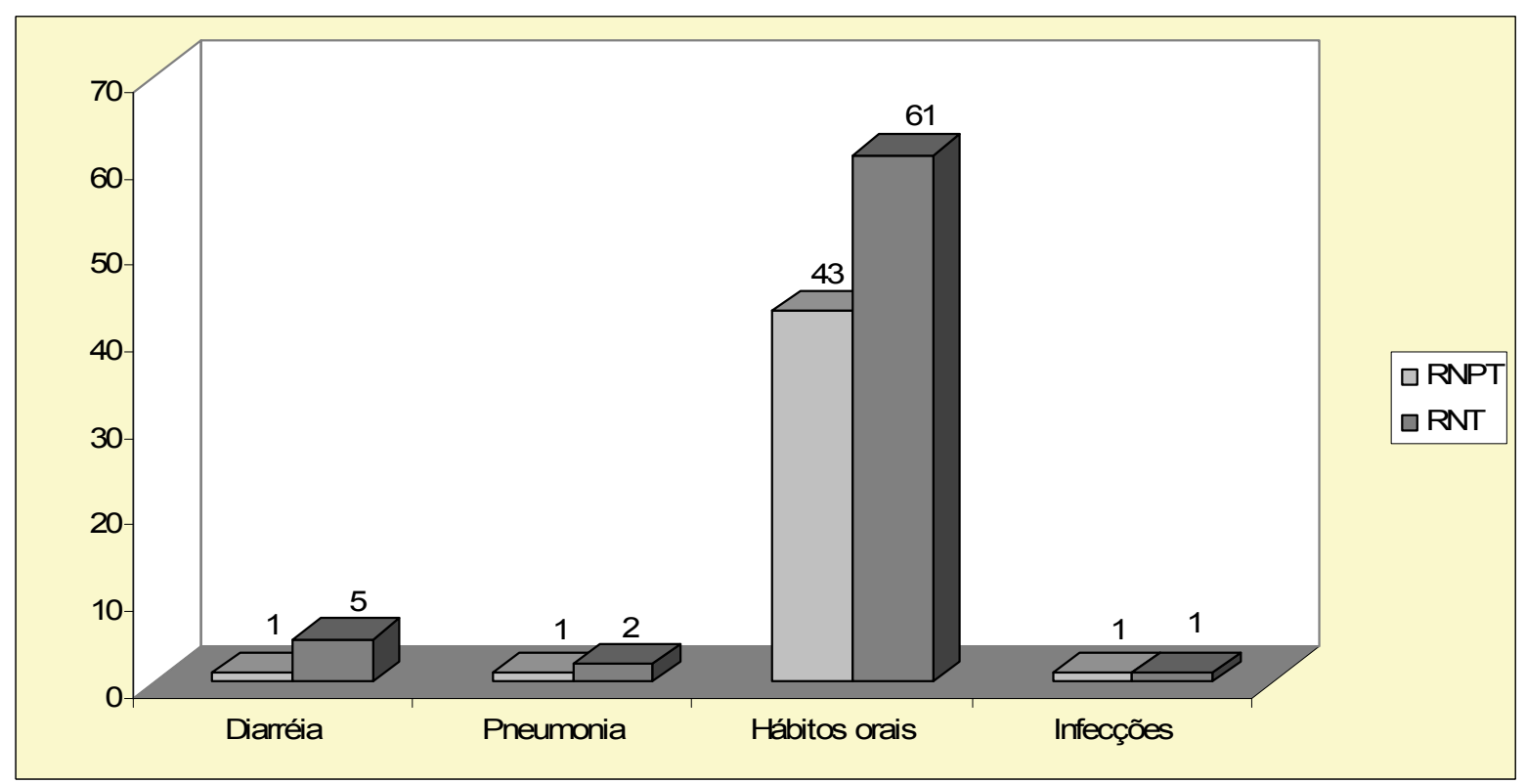

Figura 1 - Causas do desmame precoce

As causas e consequências do desmame precoce foram avaliadas nos 106 pacientes que tiveram desmame precoce e as principais causas do desmame estavam relacionadas a fatores educacionais e culturais. 


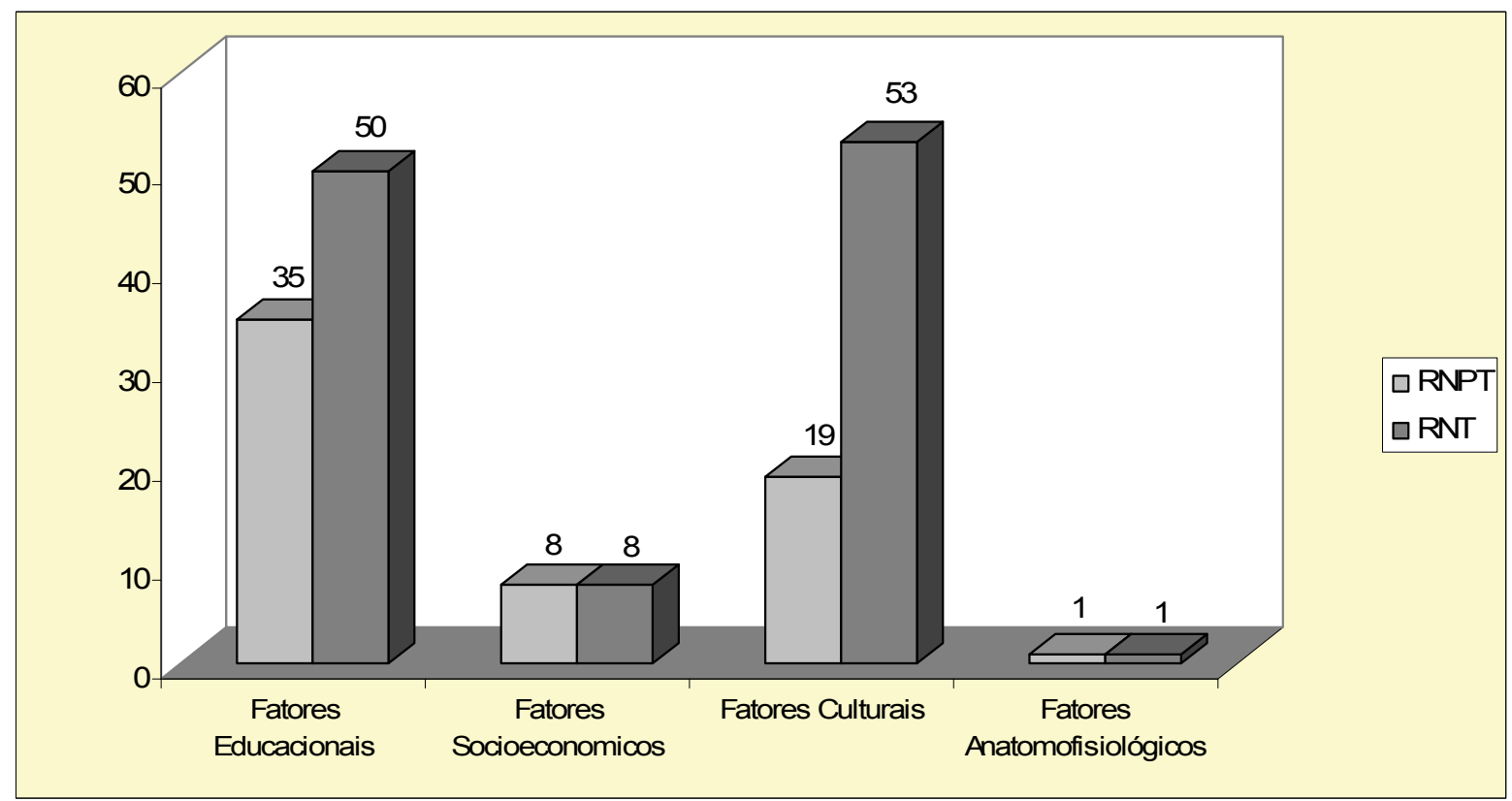

Figura 2 - Distribuição de frequências das consequências do desmame precoce

Das 106 crianças que apresentaram desmame precoce, somente uma criança não apresentou qualquer consequência quanto ao desmame precoce.

\section{DISCUSSÃO}

No presente estudo, o peso ao nascimento dos recém nascidos a termo e o dos recém nascidos prematuros, das variáveis estudadas, foi a única que apresentou significância estatística, valores estes que podem ser vistos na Tabela 1.

Em seu estudo, o autor, afirma que o baixo peso ao nascer é uma das variáveis associadas ao desmame precoce ${ }^{9}$. Em contrapartida, outros autores em suas pesquisas observaram que 0 peso ao nascer não teve relação estatística com o aleitamento materno ${ }^{10,11}$. Em seus estudos, pesquisadores afirmam que a prematuridade e o baixo peso ao nascimento são os fatores de risco para o desenvolvimento infantil, ou seja, quanto menor o peso maior a probabilidade de ocorrência de morbidade na infância ${ }^{12,13}$.

No tocante ao número de consultas de pré-natal, neste estudo houve um menor número de consultas entre as mães de crianças prematuras, dado este esperado, uma vez que o parto prematuro diminuiu o tempo de gestação e consequentemente o número de consultas pré-natais. Estudo refere que $98 \%$ das mães pesquisadas realizaram o pré-natal, porém não foi possível quantificar o número de consultas realizadas por cada mãe ${ }^{14}$.
Quanto ao tempo de uso de sonda para alimentação e o tempo de $\mathrm{O}_{2}$ das crianças prematuras, pois poucas crianças a termo fizeram uso de sonda $\mathrm{e}_{2}$ e se o fizeram foi por curto período de tempo. Dentre tais características o tempo de internação das crianças nascidas prematuras foi de 38,2 dias, enquanto que o das crianças nascidas a termo foi de 3,3 dias. Evidentemente o tempo de internação das crianças nascidas prematuras foi maior que o das crianças a termo, diferença esta já esperada, pois as crianças nascidas de parto prematuro, em sua maioria necessitam permanecer em incubadoras, em ventilação mecânica e/ou fototerapia e fazer uso de sondas para alimentação, de medicações, necessitam de cuidados especiais e essenciais para sua sobrevivência. Estudo encontrou uma média de internação de 15,6 dias para crianças nascidas prematuras, porém ela não incluiu em sua pesquisa as crianças pequenas para idade gestacional ao contrário de nosso estudo ${ }^{14}$.

$\mathrm{Na}$ presente pesquisa, o tempo médio de uso de sonda das crianças prematuras, foi de 26,7 dias, variando de 2 a 105 dias e 0 de $\mathrm{O}_{2}$ sofreu variação de 0 até 88 dias e teve uma média de 10 dias. Pesquisa desenvolvida identificou que $90 \%$ dos RNPT receberam oxigenioterapia por 2,8 dias, porém não analisou o tempo de uso de sonda ${ }^{14}$. $\mathrm{O}$ uso prolongado de sonda além de provocar uma longa permanência hospitalar traz prejuízos ao desenvolvimento de órgãos fonoarticulatórios e que existe um número razoável de crianças em fonoterapia por terem feito uso de sonda por vários 
meses, pois o uso prolongado de sonda pode levar ao atraso na coordenação dos movimentos de sucção e deglutição, pode haver hipersensibilidade oral devido a pouca ou nenhuma experiência do alimento na boca durante os primeiros meses de vida e consequentemente rejeição da alimentação por via oral, pode interferir no desenvolvimento motor oral do bebê e trazer alterações não só para as funções, mas também para os órgãos fonoarticulatórios ${ }^{15-17}$.

$\mathrm{Na}$ Tabela 2 são encontrados os dados a respeito do tempo de aleitamento materno e observou-se que $66,3 \%$ das mães entrevistadas amamentaram seus filhos por mais de 180 dias e que houve uma evidência maior e estatisticamente significante $(p=0,003)$ nas crianças prematuras $(78,0 \%)$, quando comparado com o tempo de aleitamento materno de crianças nascidas a termo (55,5\%). Ao mesmo tempo, as crianças que não foram amamentadas ou as que foram até 30 dias foi maior no grupo de RNT (16,7\%).

Em outra pesquisa foi observado que o tempo de aleitamento materno até os 30 primeiros dias de vida ocorreu em $98,1 \%$ dos casos e no sexto mês houve uma queda para $70,1 \%{ }^{9}$. Em outro trabalho a duração mediana do aleitamento materno exclusivo foi de 24 dias, do aleitamento predominante de 77 dias e do aleitamento total de 112 dias ${ }^{11}$.

Estudo realizado observou que as prevalências estimadas de aleitamento materno para o Brasil foram $87,3 \%$ aos 30 dias, de $77,5 \%$ aos 120 dias e de $68,6 \%$ aos 180 dias e do aleitamento materno exclusivo, nas mesmas idades, de $47,5 \%, 17,7 \%$ e $7,7 \%$, respectivamente ${ }^{18}$.

Em uma pesquisa, o aleitamento materno predominante ocorreu em $60,3 \%$ doas casos aos 6 meses $^{15}$. Em outra pesquisa a mediana foi de 98 dias para o AME e superior a 365 dias para o aleitamento materno ${ }^{19}$.

Com relação ao tempo de aleitamento materno exclusivo, a Tabela 3 , aponta que também houve diferença estatisticamente significante entre os grupos $(p=0,023)$ quanto ao aleitamento materno exclusivo. $\mathrm{O}$ grupo de crianças prematuras permaneceu maior tempo em aleitamento materno exclusivo que o grupo de crianças nascidas a termo, e, até os 30 primeiros dias de vida $15,8 \%$ das crianças prematuras e $31,1 \%$ das crianças nascidas a termo não estavam em aleitamento materno exclusivo.

Os RNT estão prontos para sugar e deveriam ter maiores taxas de aleitamento materno e de aleitamento materno exclusivo, porém apenas 55,5\% deles foram amamentadas por 6 meses ou mais e destas apenas 33,3\% tiveram aleitamento materno exclusivo.
A explicação para o ocorrido deve-se ao trabalho desenvolvido pela equipe multidisciplinar que assistiu o prematuro, sua mãe e familiares, orientando-os e proporcionando-lhes o suporte necessário para transpor as dificuldades encontradas, atuando como fator de proteção ao aleitamento materno ${ }^{20}$. Como também, devido à intervenção fonoaudiológica que promoveu a adequação das estruturas e funções estomatognáticas dos RNPT, favorecendo a apropriação e coordenação das funções envolvidas no processo de alimentação, favorecendo o início da amamentação no peito materno e consequente alta hospitalar.

Pesquisador desenvolveu um estudo e em seus achados, o tempo de aleitamento materno exclusivo dos lactentes até 1 mês foi de $64,8 \%$ e aos 6 meses esse valor caiu para 9,6\%. Para o aleitamento materno, a prevalência no primeiro mês foi de $98,1 \%$ e para o sexto mês de $70,1 \%$.

Em relação ao aleitamento materno exclusivo, estudo mostrou que dos 99 RNT, 66 receberam aleitamento materno exclusivo e dos 101 RNPT, 34 receberam aleitamento materno exclusivo durante a internação ${ }^{14}$.

Um dado que também deve ser considerado é que até 30 dias de vida a maioria dos RNPT ainda não tinha estabilizado seu quadro clínico, uma vez que o tempo médio de internação deste foi de 38,2 dias, porém ainda sim o desmame foi maior no grupo de crianças a termo, uma vez que a mãe precisa ser orientada e apoiada durante este período, pois há a adaptação mãe-bebê e onde são encontradas as maiores dificuldades para a amamentação (lesões nos mamilos, ingurgitamento mamário, desorganização do sono do bebê, maior cansaço e recuperação materna, principalmente do parto cirúrgico). Também, 8,7\% das mães pesquisadas realizaram o desmame durante o intervalo de 91 a 120 dias, período onde há o término da licença maternidade e onde as mesmas têm que retornar ao trabalho fora do lar. Não encontrou relação entre causa do desmame e trabalho materno ${ }^{21}$.

No estudo atual, das crianças que tiveram desmame precoce, foram verificadas as causas do desmame, sendo que a maior parte dos desmames ocorreram por fatores educacionais e por fatores culturais (Tabelas 4 e Figura 1). Em outra pesquisa as mães relataram como causas do desmame precoce além problemas relacionados à "falta de leite", "leite fraco" (fatores educacionais, relacionadas à falta de informação e orientação) a influência de fatores culturais (uso de chupeta e mamadeira, crenças) $)^{13,22}$.

Os fatores associados ao aleitamento materno e ao aleitamento materno exclusivo foram analisados em dois estudos e os autores observaram o uso de 
chupeta e mamadeira como fatores culturais que levam ao desmame precoce $e^{9,23,24}$.

As consequências do desmame, na atual pesquisa, podem ser observadas na Figura 2. Também foi observado em nossa pesquisa 5 casos associados com diarréia, 3 a pneumonia e 2 a infecções.

Outro autor aponta algumas consequências do desmame precoce, a morte por diarréia, infecções respiratórias agudas e outras doenças infecciosas, são 20 vezes maiores em crianças desmamadas precocemente $^{25,26}$. Pesquisa verificou que houve uma associação estatisticamente significante entre o desmame e o uso de chupeta ${ }^{27}$ e mamadeira ${ }^{9}$. Crianças amamentadas até um mês de idade, que usavam chupeta, tiveram uma chance 2,8 vezes maior de serem desmamadas até o sexto mês ${ }^{27}$.

Crianças com até 2 anos de idade e amamentadas por menos de 4 meses apresentaram 2 vezes mais chances de apresentar infecções de vias aéreas superioras, e as crianças pré-termo tinham menor chance de ter pneumonia quando amamentadas exclusivamente por 4 meses ou mais ${ }^{27,28}$.

Ao ponderar a respeito do leite materno, pesquisa descreve que o mesmo possui enzimas, vitaminas, água, sais minerais, anticorpos, hormônios, lisozimas e imunoglobulinas que defendem a criança de infecções ${ }^{10}$. Por sua vez, a introdução precoce de alimentos complementares está associada ao aumento da morbidade e mortalidade infantil, devido a menor ingesta de anticorpos e imunoglobulinas contidos no leite materno e ao maior risco de contaminação dos alimentos ofertados às crianças ${ }^{29}$.

Os benefícios do aleitamento materno são incomparáveis, pois reduz a mortalidade e morbidade infantil, a incidência e gravidade de doenças como anemia, diarréias, infecções respiratórias, otites médias, infecções urinárias e doenças alérgicas e que as crianças menores que seis meses que não mamavam apresentaram uma chance $64,0 \%$ a mais para a diarréia que aquelas amamentadas. Quando comparadas com as que mamavam exclusivamente, houve um aumento desse percentual para $82,0 \%$ entre as não amamentadas ${ }^{30-32}$.

Outros autores apontam algumas consequências do desmame precoce ${ }^{33}$, a morte por diarréia, infecções respiratórias agudas e outras doenças infecciosas, são 20 vezes maiores em crianças desmamadas precocemente ${ }^{28}$. Estudo verificou que houve uma associação estatisticamente significante entre o desmame e o uso de chupeta e mamadeira ${ }^{9}$. Pesquisador encontrou em sua pesquisa que as crianças amamentadas até um mês de idade, que usavam chupeta, tiveram uma chance 2,8 vezes maior de serem desmamadas até o sexto mês, pois há uma menor estimulação da mama e menor produção de leite ${ }^{28}$.

O aleitamento materno não é somente biológico, mas uma associação de fatores históricos, educacionais, sociais, anatomofuncionais, culturais e psicológicos. Não obstante, não satisfaz apenas informar, faz-se necessário mudar crenças, derrubar tabus da mulher e da sociedade em geral. Talvez esse seja o motivo das taxas de aleitamento materno estarem ainda longe do preconizado pela OMS. Faz-se necessária uma intervenção em diversas áreas, como a mudança de conceitos arraigados no cerne de mulheres no decorrer de anos, passados de geração em geração. Essas mães/ mulheres precisam ser apoiadas, melhor orientadas e assistidas.

A amamentação é também a responsável por uma maior economia para família e para o Estado, pois não é necessário comprar leite artificial e demais fórmulas lácteas para suprir as necessidades decorrentes do desmame precoce. Políticas voltadas à promoção, proteção e incentivo ao aleitamento materno e a alimentação complementar saudável após os seis meses de vida devem ser estimuladas, com 0 intuito de proporcionar às crianças e à suas mães uma vida mais saudável e com qualidade.

Deve ser ressaltada a importância do trabalho de uma equipe multidisciplinar no atendimento ao prematuro e sua mãe, cabendo ao fonoaudiólogo intervir o mais precocemente possível nessa díade com o propósito de adequar a funções de sucção, deglutição e respiração, favorecendo o início da amamentação ao prematuro, reduzindo o tempo de hospitalização e reduzindo os custos para o Estado.

Mediante ao que foi exposto, ao menos resta a satisfação de saber que as taxas de aleitamento materno estão aumentando, mostrando que algumas ações estão sendo desenvolvidas como: a criação de Enfermarias Canguru para assistência de crianças prematuras e de baixo peso, a implantação de Hospitais Amigos da Criança, a formação de grupos de apoio e incentivo ao aleitamento materno, o desenvolvimento de pesquisas em Fonoaudiologia voltadas para neonatos normais e de risco, com a finalidade de prestar melhor assistência às crianças, suas mães e familiares. Estas intervenções estão sendo desenvolvidas para seguir as orientações da Organização Mundial de Saúde e para minimizar as consequências que o desmame precoce traz, a curto e em longo prazo, tanto para as mães - que são acolhidas, assistidas e informadas dos cuidados para consigo e para com seu filho -, quanto para os recém nascidos que são amamentados por mais tempo diminuindo 
o risco de morte e de agravos de saúde na infância por desnutrição, diarréia, infecções e pneumonia.

No entanto, existe muito a ser feito para intervir no aumento desses percentuais. Realizar projetos junto aos órgãos públicos de saúde que favoreçam a amamentação, formar grupos de apoio e incentivo ao aleitamento materno, capacitar os profissionais de saúde que já lidam com a amamentação, realizar campanhas educativas e divulgar as ações em prol do aleitamento materno, criar Bancos de Leite Humano e estimular as mães a doarem o leite materno que é importantíssimo para o bebê e principalmente para o prematuro. Além disso, faz-se necessário provar a população que amamentar é possível para a maioria das mulheres, salvo alguns casos onde o aleitamento materno é contra-indicado, talvez essa seja a tarefa mais difícil. Mostrar que, de fato, o aleitamento materno é a forma mais natural e mais saudável de se alimentar uma criança.

Os profissionais de saúde devem ser comunicadores, educadores e formadores de opinião em saúde ${ }^{34}$. Devem desenvolver no indivíduo a responsabilidade no cuidado com a sua saúde e de sua família. È evidente que esta não é uma prática fácil, haja vista a extensão de nosso País e por ser o Brasil repleto de diversidades. Contudo, a promoção do aleitamento materno, deve ser incluída entre as ações prioritárias de saúde ${ }^{35}$, e os fatores supracitados justificam a necessidade de programas de incentivo ao aleitamento materno em cada região ${ }^{36}$, para que as taxas de aleitamento materno e de aleitamento materno exclusivo preconizadas pela Organização Mundial de Saúde sejam uma realidade para todos.

\section{CONCLUSÃO}

O tempo de aleitamento materno e de aleitamento materno exclusivo dos prematuros foi superior aos valores encontrados para as crianças nascidas a termo. As crianças nascidas de parto prematuro apresentaram uma média de 121,6 dias para o aleitamento materno exclusivo e as nascidas a termo de 96,3 dias havendo diferença estatística entre os grupos. Mais da metade das crianças estudadas foram desmamadas antes dos 6 meses de vida.

Quando comparados RNT com RNPT a variável peso ao nascer apresentou diferença significante entre os grupos. Os fatores educacionais e culturais foram as principais causas do desmame precoce e quase todas as crianças desmamadas precocemente tiveram como consequência do desmame o uso de chupeta e mamadeira.

\begin{abstract}
Purpose: to determine the benefits of exclusive breastfeeding. However, only a minority of women engage in breastfeeding practices. This research is aimed to establish the time of exclusive breastfeeding as for preterm babies and observe whether they have a different level of exclusive breastfeeding in relation to the level achieved by the city of Maceió at the I and II PPAM-CDF. Additionally, other goals are to compare the breastfeeding and exclusive breastfeeding types of preterm and term newborns and determine the causes of early weaning and its consequences. Method: this is a field investigation with exploratory and longitudinal nature on the prevalence of exclusive breastfeeding of preterm and term babies. Results: as for the percentage of breastfeeding and exclusive breastfeeding of preterm babies at 6 months old. There were significant differences in the birth weight variable. The causes for the early weaning, we found to be educational factors, followed by cultural factors. As a consequence of early weaning, the practice of oral habits occurred in $98.1 \%$ of the children. Conclusion: preterm children remained more time in breastfeeding and exclusive breastfeeding than the term babies. The educational and cultural factors were the main causes for the early weaning, and the consequence was the use of pacifier and bottle.
\end{abstract}

KEYWORDS: Breast Feeding; Weaning; Infant, Newborn 


\section{REFERÊNCIAS}

1. Casagrande L, Ferreira FV, Hahn D, Unfer DT, Praetzel JR. Aleitamento natural e artificial e o desenvolvimento do sistema estomatognático. Rev. Fac. Odontol., 2008;49(2):11-7.

2. Monson MRR; Becker EA; Witte E; Almeida JAG; Filho JBL; Teles MDG; Goldemberg P. Revisão e avaliação das ações nacionais para implementação do código internacional de comercialização dos substitutos do leite materno. Brasília-DF: Gráfica Modelo, 1991, 43p.

3. Simon VGN, Souza JMP, Souza SB. Aleitamento materno, alimentação complementar, sobrepeso e obesidade em pré-escolares. Rev Saúde Pública. 2009;43(1):60-9.

4. Spyrides MHC, Struchiner CJ, Barbosa MTS, Kac G. Amamentação e crescimento infantil: um estudo longitudinal em crianças do Rio de Janeiro, Brasil, 1999/2001. Cad. Saúde Pública, Rio de Janeiro. 2005;21(3):756-66.

5. Parizzoto J, Zorzi NT. Aleitamento Materno: fatores que levam ao desmame precoce no município de Passo Fundo, RS. O Mundo da Saúde São Paulo. 2008;32(4):466-74.

6. Gianini NOM. Leite materno e prematuridade. In: Rego JD. Aleitamento materno. 2a ed. São Paulo: Atheneu, 2006, pp. 261-83.

7. Araújo, MFM. Situação e perspectivas do aleitamento materno no Brasil. In: Carvalho, MR; Tamez RN. Amamentação bases científicas para a prática profissional. Rio de Janeiro: Guanabara Koogan, 2002, pp. 1-10.

8. Brasil. Ministério da Saúde. Pesquisa de Prevalência de Aleitamento Materno nas Capitais Brasileiras e no Distrito Federal PPAM-CDF. Ministério da Saúde, 1999.<http:// www.bvsam.cict.fiocruz.br/gotadeleite/01/arqs/ pesqnacprevalencia99.ppt>Acesso em $14 \mathrm{de} \mathrm{fev.}$ 2007.

9. Audi CAF, Corrêa AMS, Latorre MRDO. Alimentos complementares associados ao aleitamento materno e ao aleitamento materno exclusivo em lactentes até 12 meses de vida em Itapira, São Paulo, 1999. Rev. bras. Saúde matern. infant., 2003;3(1):85-93.

10. Santos VFS, Soler ZASG, Azoubel R. Alimentação de crianças no primeiro semestre de vida: enfoque no aleitamento materno exclusivo. Rev. Bras. Matern. Infant., Recife. 2005;5(3):283-91. 11. Vasconcelos MGL, Lira PIC, Lima MC. Duração e fatores associados ao aleitamento materno em crianças menores de 24 meses de idade no estado de Pernambuco. Rev. Bras. Saúde Matern. Infant., Recife. 2006;6(1):99-105.
12. Baptista GH, Andrade AHHKG, Giolo SR. Fatores associados à duração do aleitamento materno em crianças. Cad. Saúde Pública, Rio de Janeiro, 2009;25(3):596-604.

13. Ramos HAC, Cuman RKN. Fatores de risco para prematuridade: pesquisa Documental. Esc Anna Nery Rev Enferm. 2009;13(2):297-304.

14. Yagi RGR. Alimentação de recém-nascidos e lactentes a termo e prematuros atendidos em um Hospital Escola [Tese]. São José do Rio Preto: Faculdade de Medicina de São José do Rio Preto; 2005. Available from: http://bdtd.famerp.br//tde busca/arquivo.php?codArquivo $=30$.

15. Penalva O, Schwartzman JS. Descriptive study of the clinical and nutritional profile and follow-up of premature babies in a Kangaroo Mother Care Program. J Pediatr (Rio J). 2006;82(1):33-9.

16. Proença MG, Hernandez MH. Desenvolvimento sensório-motor da sucção e deglutição em recém nascidos pré-termo com alterações genéticas ou com problemas outros que exijam alimentação por sonda ou estomia. In: Sistema sensório motor oral: perspectiva de avaliação e terapia. São Paulo: EDUC, 1987, pp. 86-100.

17. Xavier C. Intervenção fonoaudiológica em bebês de risco. In: Ferreira LP. Tratado de Fonoaudiologia. São Paulo: Roca, 2004, pp. 415-38.

18. Scochi, CGS, Ferreira FY, Góes FSN, Fujinaga, $\mathrm{Cl}$, Ferecini GM, Leite AM. Alimentação láctea e prevalência do aleitamento materno em prematuros durante internação em um hospital amigo da criança de ribeirão preto-sp, Brasil. Cienc Cuid Saude. 2008;7(2):145-54.

19. Ramos CV, Almeida JAG, Saldiva SRDM, Luciana Maria Ribeiro Pereira, Alberto NSMC, Teles JBM, Pereira TG. Prevalência do Aleitamento Materno Exclusivo e os fatores a ele associados em crianças nascidas nos Hospitais Amigos da criança de Teresina - Piauí. Epidemiol. Serv. Saúde, Brasília. 2010;19(2):115-24.

20. Braga DF, Machado MMT, Bosi MLM. Amamentação exclusiva de recém-nascidos prematuros: percepções e experiências de lactantes usuárias de um serviço público especializado, 2008;Rev. nutr;21(3):293-302.

21. Brasileiro AA, Possobon RF, Carrascoza $\mathrm{KC}$, Ambrosano GMB, Moraes ABA. Impacto do incentivo ao aleitamento materno entre mulheres trabalhadoras formais. Cad. Saúde Pública, 2010, Rio de Janeiro, 26(9):1705-13.

22. Castro IRR, Engstrom EM, Cardoso LO, Damião JJ, Rito RVFV, Gomes MASM.Tendência temporal da amamentação na cidade do Rio de Janeiro: 1996-2006. Rev. Saúde Pública [online], 2009;43(6):1021-9. 
23. Araújo OD, Cunha AL, Lustosa LR, Nery IS, Mendonça RCM, Campelo SMA. Aleitamento materno: fatores que levam ao desmame precoce. Rev. bras. enferm., 2008;61(4):487-92.

24. França MCT, Giugliani ERJ, Oliveira LD, Weigert EML, Santo LCE, Köhler CV, Bonilh ALL. Uso de mamadeira no primeiro mês de vida: determinantes e influência na técnica de amamentação. Rev Saúde Pública, 2008; 42(4):607-14.

25. Fuchs SC, Victora CG. Risk and prognostic factors for diarrheal disease in Brazilian infants: a special case-control design application. Cad. Saúde Pública, 2002;18(3):773-82.

26. Silveira FJF, Lamounier JA. Fatores associados à duração do aleitamento materno em três municípios na região do Alto Jequitinhonha, Minas Gerais, Brasil. Cad. Saúde Pública. 2006;22(1):69-77.

27. Soares MEM, Giugliani ERJ, Braun ML, Salgado ACN, Oliveira AP, Aguiar PR. Uso de chupeta e sua relação com o desmame precoce em população de crianças nascidas em Hospital Amigo da Criança. J. Pediatr (Rio J). 2003;79(4):309-16.

28. Carvalho CF, Silva MGF. Avaliação do desmame precoce e suas implicações infecciosas nas crianças atendidas no ambulatório de um hospital terciário. Arq Ciênc Saúde, 2005;12(3):129-32.

29. França GVA, Brunken GS, Silva SM, Escuder MM, Venâncio SI. Determinantes da amamentação no primeiro ano de vida em Cuiabá, Mato Grosso. Rev. Saúde Pública, 2007; 41(5):711-8.
30. Vieira GO, Silva LR, Vieira TO. Alimentação infantil e morbidade por diarréia. J Pediatr (Rio J) 2003;79(5):449-54.

31. Carrascoza KC, Possobon RF, Tomita ML, Moraes ABA. Consequences of bottle-feeding to the oral facial development of initially breastfed children. J Pediatr (Rio J), 2006; 82(5):395-7.

32. Levy L, Bértolo H. Manual de Aleitamento Materno. Comité Português para a UNICEF/ Comissão Nacional Iniciativa Hospitais Amigos dos Bebés, Edição Revista de 2008, 43p.

33. Costa CN, Lima GRS, Jorge RM, Malta RACG, Nemr K. Efetividade da intervenção fonoaudiológica no tempo de alta hospitalar do recém-nascido pré-termo. Rev CEFAC. 200; 9(1):72-8.

34. Santana MCCP, Goulart BNG, Chiari BM, Melo AM, Silva EHAA. Aleitamento materno em prematuros: atuação fonoaudiológica baseada nos pressupostos da educação para promoção da saúde. Ciência \& Saúde Coletiva. 2010;15(2):411-7. 35. Fujimori E, Nakamura E, Gomes MM, Jesus LA, Rezende MA. Issues involved in establishing and maintaining exclusive breastfeeding, from the perspective of women attended at a primary healthcare unit. Interface - Comunic., Saude, Educ. 2010;14(33):315-27.

36. Almeida CC, Scochi MJ, Souza RKT, Carvalho WO. Prevalência de aleitamento materno antes e após a implantação de um programa de redução de morbimortalidade infantil, no município de Campo Mourão (PR). Ciência \& Saúde Coletiva, 2010;15(2):575-83.
http://dx.doi.org/10.1590/S1516-18462012005000055

RECEBIDO EM: 08/06/2011

ACEITO EM: 05/12/2011

Endereço para correspondência:

Waléria Ferreira da Silva

Rua Amazonas, no 22, BI “A”, apto 303

Feitosa - Maceió - AL

CEP: $57043-450$

E-mail: ferreira.waleria@gmail.com 